\title{
Vitamin B1-Substitution schützt Nerven und Gefäße
}

\begin{abstract}
— Diabetiker weisen häufig einen ausgeprägten Mangel an Vitamin B1 (Thiamin) auf. Dieses Defizit ist an der Pathogenese der diabetischen Neuropathie, einer Folgeerkrankung des Diabetes mellitus, beteiligt. Im Vitamin B1-Mangelzustand akkumulieren schädliche Verbindungen des Glukosestoffwechsels und entfalten pathogene Wirkungen an den Gefäßen.

Zudem verstärkt Vitamin B1- bzw. ThiaminMangel die Auswirkungen von oxidativem Stress und fördert entzündliche Reaktionen. Folgeerkrankungen wie Neuro-, Retinound Nephropathie sind dann möglich, erklärte Professor Karlheinz Reiners von der Neurologischen Klinik des Hermann-JosefKrankenhauses Erkelenz.
\end{abstract}

Im peripheren Nervensystem begünstigt Vitamin B1-Mangel die Entwicklung einer Polyneuropathie. Im Zentralnervensystem kann der Mangel zu einer toxischen Mittelhirnschädigung wie der Wernicke-Enzephalopathie oder zu einer Neurotransmitterstörung wie der Korsakow-Psychose führen. Kompensiert werden kann dies durch die Substitution mit hoch bioverfügbarem Thiamin, erklärte Reiners. Die fettlösliche Thiamin-Vorstufe Benfotiamin (milgamma ${ }^{\circledR}$ protekt) erzielt signifikant höhere Konzentrationen an aktivem Thiamin-Diphosphat (TDP) im Plasma und in den Zellen als wasserlösliche Thiamin-Derivate. Bei der Behandlung der diabetischen Neuropathie ist das kausal wirksame Benfotiamin neben der normnahen Blutzuckereinstellung ein effektiver Therapieansatz. Eine symptomatische Therapie mit Analgetika sei dagegen aufgrund ihrer Nebenwirkungen auf Dauer keine gute Lösung, so Reiners.

Durch den frühzeitigen Ausgleich des Vitamin B1-Defizits durch Benfotiamin werden pathogene Prozesse im diabetischen Stoffwechsel kompensiert. Bei regelmäßiger Einnahme schützt Benfotiamin Nerven und Gefäße vor schädlichen Abbauprodukten des Glukosestoffwechsels und kann Neuropathie-Symptome wie Schmerzen, Kribbeln, Brennen oder Taubheit in den Füßen lindern. Dass Benfotiamin diese Symptome deutlich lindern kann, konnte durch Studien belegt werden.

(djb)

Pressekonferenz „Diabetes! Hören Sie auf Ihre Füße? Diabetische Neuropathie im Fokus - Aktuelles aus Wissenschaft und Praxis"; Hamburg, 24. Mai 2017 (Veranstalter: Wörwag Pharma $\mathrm{GmbH} \&$ Co.KG)

\section{Technologiegestützte Pflege Mit Sensorik und Robotik dem Fachkräftemangel entgegenwirken}

— Die Technik kann den Menschen nicht ersetzen - vor allem nicht in der Pflege. Aber Fachkräftemangel und Nachwuchssorgen erfordern innovative Ansätze. Neue Technologien können dabei die Pflege sinnvoll unterstützen. Die demographische Entwicklung in Deutschland ist der Motor für innovative Ideen im Gesundheitssektor. Prognosen sagen voraus, dass es im Jahr 2030 vier Millionen Pflegebedürftige geben wird. Der Fachkräftemangel in der Pflege ist jedoch heute schon allgegenwärtig. „Wenn wir es schaffen, Daten zu vernetzen und auszuwerten, gewinnen wir mehr Zeit für die Pflege", erklärte auf dem Hauptstadtkongress für Gesundheitsberufe Dr. Florian Frensch von Philips. Der Medikamentendispenser Medido von Philips beispielsweise ermöglicht es älteren Menschen, ihre Selbstständigkeit zu erhalten. Mit jedem Medikament, das sie einnehmen müssen, steigt die Wahrscheinlichkeit für Unregelmäßigkeiten, eigenmächtige Dosisänderungen und Therapieabbrüche. Medido kann die Patienten bei ihrer Medikamenteneinnahme zu Hause unterstützen: Der Dispenser gibt die individuell verblisterten Medi- kamente zu voreingestellten Zeitpunkten aus und erinnert an die Einnahme durch ein Licht- und Tonsignal. Sollte der Patient die Einnahme nicht bestätigen, informiert Medido den ambulanten Pflegedienst oder die Angehörigen.

Ergänzend dazu stellte Frensch das CareSage-System vor. Dieses System kann das Risiko einer Krankenhauseinweisung bis zu 90 Tage im Voraus ermitteln. Der innovative Algorithmus basiert auf den Hausnotrufsystemen von Philips und kombiniert deren Daten mit Informationen aus der individuellen Krankengeschichte. Damit bietet das System die Möglichkeit einer gezielten Risikoprävention und Versorgungssteuerung in der ambulanten Pflege. Viele Krankenhausaufenthalte älterer, chronisch kranker Patienten können so verhindert werden. Insgesamt sorgen diese Lösungsvorschläge für eine höhere Lebensqualität und ein stärkeres Sicherheitsgefühl bei den Patienten. Im Rahmen der Veranstaltung stellte Prof. Elena Giannoulis von der Freien Universität Berlin ihr Forschungsprojekt zur bereits in Japan angewandten Robotik in der Pflege vor. Dort lebt der Emotionsrobo- ter Pepper schon jetzt in Familien und Seniorenheimen. Für deutsche Ohren ist das noch Zukunftsmusik. Doch die Experten auf dem Podium waren sich einig: Roboter in der Pflege sind ein realistisches Zukunftsmodell - zumindest in bestimmten Bereichen.

„Pflege innovativ: Wie Sensorik, Robotik und das Internet die Pflege verändern"; Hauptstadtkongress Medizin und Gesundheit, 20. Juni 2017.

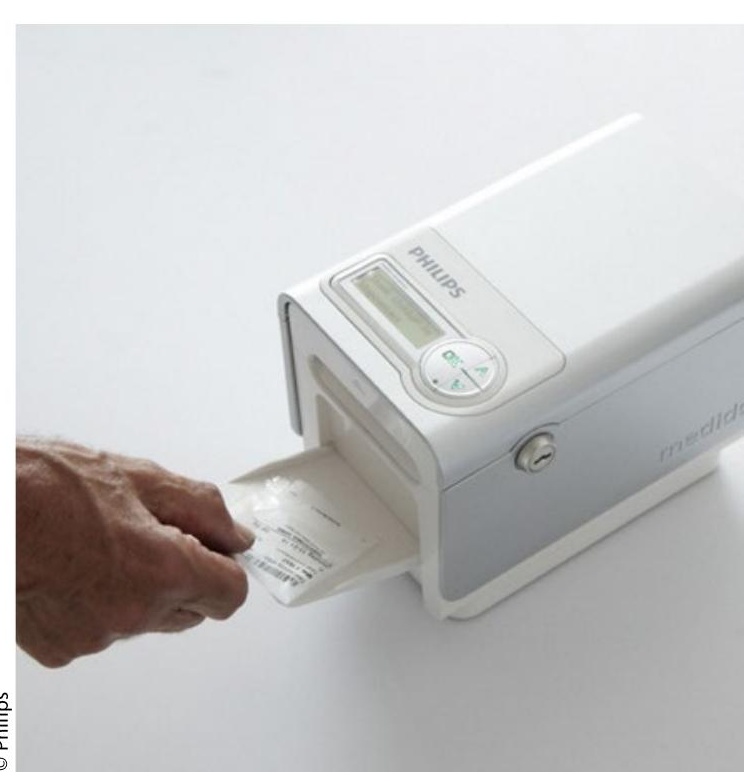

